\title{
A probabilistic approach for validation of advanced driver assistance systems*
}

\author{
O.J. Gietelink, B. De Schutter, and M. Verhaegen
}

If you want to cite this report, please use the following reference instead: O.J. Gietelink, B. De Schutter, and M. Verhaegen, "A probabilistic approach for validation of advanced driver assistance systems," Proceedings of the 8th TRAIL Congress 2004 - A World of Transport, Infrastructure and Logistics - CD-ROM, Rotterdam, The Netherlands, 17 pp., Nov. 2004.

Delft Center for Systems and Control Delft University of Technology

Mekelweg 2, 2628 CD Delft

The Netherlands

phone: +31-15-278.51.19 (secretary)

fax: $+31-15-278.66 .79$

URL: http://www.dcsc.tudelft.nl

${ }^{*}$ This report can also be downloaded via http://pub.deschutter.info/abs/04_016.html 


\section{A probabilistic approach for validation of advanced driver assistance systems}

TRAIL Research School, Delft, November 2004

\section{Authors}

ir. O.J. Gietelink ${ }^{* \dagger}$, dr.ir. B. De Schutter*, prof.dr.ir M. Verhaegen*

${ }^{*}$ Delft University of Technology, Delft Center for Systems and Control

$\dagger$ TNO Automotive 



\title{
Contents
}

\begin{abstract}
1

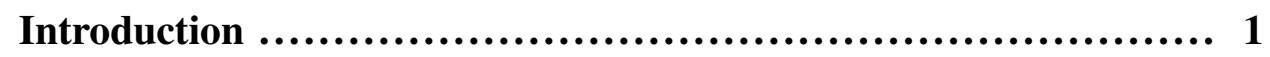

Advanced driver assistance systems ............................ 1

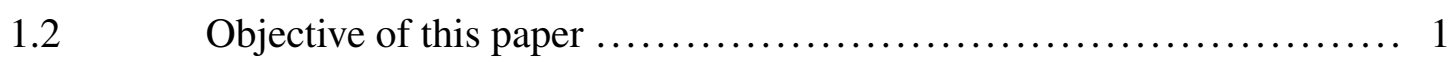

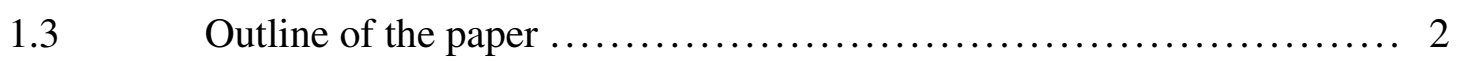

2 A case study: Adaptive cruise control $\ldots \ldots \ldots \ldots \ldots \ldots \ldots \ldots \ldots \ldots . \ldots . \ldots$

2.1 A simplified model and control law for adaptive cruise control ........ 3

2.2 Performance criteria and perturbations for ACC .................. 4

The control system validation problem........................ 4

3 A randomized algorithm for control system validation..............6

3.1 Motivation for a probabilistic approach ........................ 6

B.2 Bounded sample complexity for RAs ......................... 6

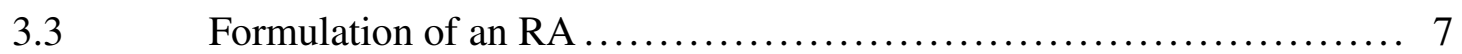

Examples of application of an RA............................ 8

3.4.1 Example 1: Uniformly distributed disturbance .................. 8

3.4.2 Example 2: Gaussian Distributed Disturbance $\ldots \ldots \ldots \ldots \ldots \ldots \ldots \ldots \ldots$

3.4.3 Example 3: Multi-dimensional disturbance ....................... 10

Characteristics properties of RAs ............................. 11
\end{abstract}

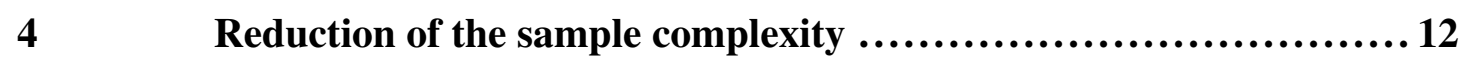

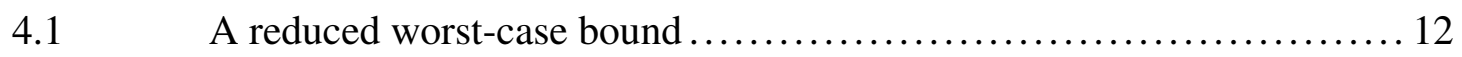

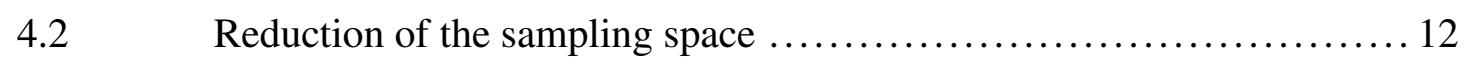

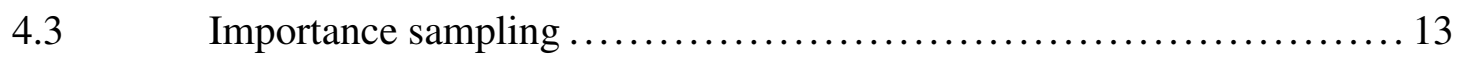

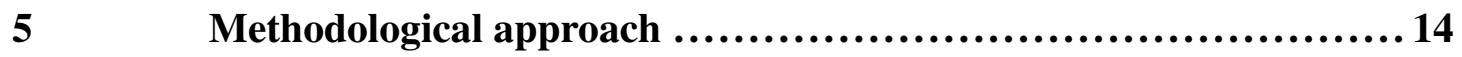

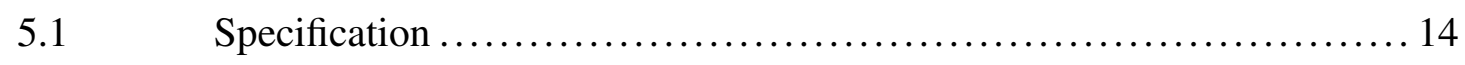

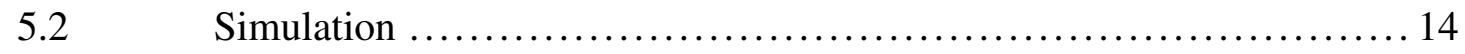

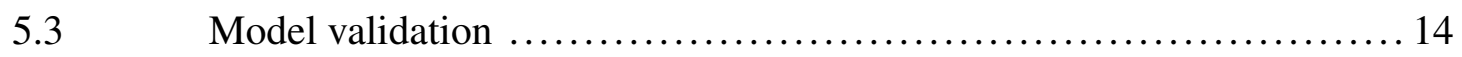


Acknowledgments . 17

References......................................................................... 18 



\begin{abstract}
This paper presents a methodological approach for validation of advanced driver assistance systems. The methodology relies on the use of randomized algorithms that are more efficient than conventional validation using simulations and field tests, especially with increasing complexity of the system. The methodology consists of first specifying the perturbation space and performance criteria. Then a minimum number of samples and a relevant sampling space is selected. Next an iterative randomized simulation is executed, followed by validation of the simulation model by hardware tests, in order to increase the reliability of the estimated performance. The concept is illustrated with some examples of a case study involving an adaptive cruise control system. The case study also points out some characteristic properties of randomized algorithms regarding the necessary sample complexity, and the sensitivity to model uncertainty. Solutions for these issues are proposed as well as corresponding recommendations for future research.
\end{abstract}

\title{
Keywords
}

Advanced driver assistance systems, intelligent vehicles, controller validation, randomized algorithms, adaptive cruise control 



\section{Introduction}

\subsection{Advanced driver assistance systems}

The increasing demand for safer passenger vehicles has stimulated the research and development of advanced driver assistance systems (ADASs) over the past decade. An ADAS typically consists of environment sensors (e.g. radar, laser, and vision sensors) and electronic control functions to improve driving comfort and traffic safety by warning the driver, or even autonomous control of actuators. State-of-the-art examples of ADASs that have already been introduced by the automotive industry are adaptive cruise control (ACC), collision warning systems, and pre-crash systems.

The demand for safety and reliability naturally increases with the increasing automation of the vehicle's driving task, since the driver must fully rely on flawless operation of the ADAS. E.g., autonomous braking in an ACC should be executed only if the distance to the preceding vehicle will otherwise become unacceptably small, or even result in a collision. In order to handle a large variety of complex traffic scenarios and disturbances, redundancy and fault-tolerance measures are often implemented. In practice, it is however difficult to choose the right control measures and to validate their effectiveness. Manufacturers thus face an increasing effort in the design and validation of ADASs in contrast to a desired shorter time-to-market.

Currently, an iterative process of simulations and prototype test drives on a test track is used for validation purposes. The simulation effort can however be unreliable due to model uncertainty, and inefficient, since a large number of simulations are necessary to achieve a representative result. Instead, a worst-case analysis is often used, which leads to a conservative control system design, thus limiting the functional performance of the ADAS. On the other hand, test drives are more reliable, but can never cover the entire set of operating conditions, due to time and cost constraints. Furthermore, test results can be difficult to analyze for controller validation and benchmarking, because traffic scenarios cannot be exactly reproduced during a test drive. It may therefore become impossible to evaluate an ADAS with guaranteed measures for the level of performance, safety, and reliability.

\subsection{Objective of this paper}

The objective of this paper is to present a methodological approach based on randomized algorithms (RAs) to provide an efficient test program in order to cover the entire set of operating conditions with a minimum number of samples. A simplified case study will be used as an illustration of this methodology for reasons of transparency. Note that the emphasis of this paper is therefore on the validation process, and not on control system design.

One validation tool in this methodology is the software tool PRESCAN, which is used in a probabilistic simulation strategy with the operating conditions chosen to be representative for the 'real' conditions. The simulation models can subsequently be validated with another tool: the VEhicle-Hardware-In-the-Loop (VEHIL) facility, TNO's tailor-made laboratory for testing ADASs. In VEHIL a real ADAS equipped vehicle is tested in a hardware-in-the-loop simulation, as shown by the working principle in Figure 1. With VEHIL the development process and more specifically the validation phase of ADASs can be carried out safer, cheaper, more manageable, and more reliable. Both 


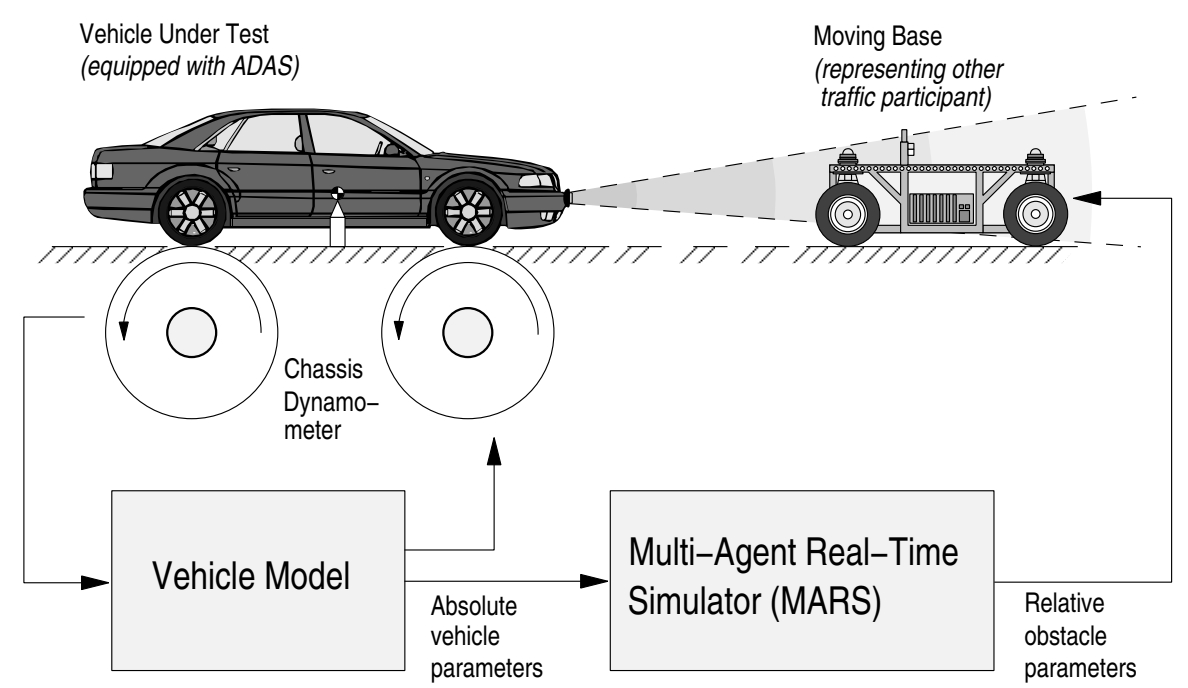

Figure 1: VEHIL working principle.

VEHIL and PRESCAN are fully described by Gietelink et al. (2004), and in this paper we restrict the discussion to the underlying validation methodology, as outlined below.

\subsection{Outline of the paper}

This paper is organized as follows. In Section 2 a simplified model of an ACC system is presented, together with its performance measures and the perturbations acting on the system. Section 3 treats the background theory of RAs that is subsequently applied to the ACC case study in a number of examples. These examples illustrate the advantages of using RAs, but also highlight some points for improvement, especially regarding the required number of samples. Section 4 discusses some possibilities for addressing this issue. Subsequently, Section 5 presents an improved methodological approach for the validation of ADASs. This methodology consists of first specifying the perturbation space and performance criteria. Then a minimum number of samples and a relevant sampling space is selected, using the reduction techniques from Section 4. Next an iterative randomized simulation is executed, followed by validation of the simulation model by hardware tests in the VEHIL facility, in order to increase the reliability of the estimated performance. Finally, Section 6 summarizes the validation approach, and discusses ongoing research activities. The added value of this paper is to connect the research areas of ADASs, RAs, and conventional methods for control system validation. 


\section{A case study: Adaptive cruise control}

\subsection{A simplified model and control law for adaptive cruise control}

Figure 2 illustrates an ACC-equipped vehicle following a lead vehicle, with their position $x$ and velocity $v$, where the subscripts ' $\mathrm{l}$ ' and ' $\mathrm{f}$ ' denote leader and follower respectively. Further defined are the distance between the two vehicles (the headway) $x_{\mathrm{r}}=x_{1}-x_{\mathrm{f}}$, the relative velocity $v_{\mathrm{r}}=v_{\mathrm{l}}-v_{\mathrm{f}}$, the desired distance $x_{\mathrm{d}}$, and the headway separation error $e_{x}=x_{\mathrm{d}}-x_{\mathrm{r}}$.

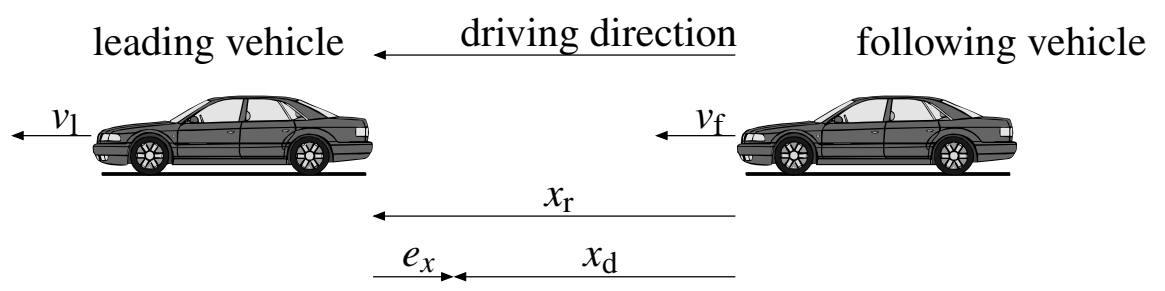

Figure 2: Two vehicles in ACC mode: one leader and one follower.

ACC tries to maintain a pre-defined velocity set-point $v_{\mathrm{cc}}$, unless a slower vehicle is detected ahead. In that case vehicle ' $\mathrm{f}$ ' is controlled to follow vehicle ' $\mathrm{l}$ ' with equal velocity $v_{\mathrm{f}}=v_{\mathrm{l}}$ at a desired distance $x_{\mathrm{d}}$. Since the ACC objective is to control the motion of a vehicle relative to a preceding vehicle, the vehicle state is chosen as $\mathbf{x}=\left[\begin{array}{ll}x_{\mathrm{r}} & v_{\mathrm{r}}\end{array}\right]^{T}$. We can describe the evolution of the systems by

$$
\dot{\mathbf{x}}=\left[\begin{array}{ll}
0 & 1 \\
0 & 0
\end{array}\right]\left[\begin{array}{l}
x_{\mathrm{r}} \\
v_{\mathrm{r}}
\end{array}\right]+\left[\begin{array}{c}
0 \\
-1
\end{array}\right] a_{\mathrm{f}}+\left[\begin{array}{l}
0 \\
1
\end{array}\right] a_{1}
$$

where the acceleration of the following vehicle $a_{\mathrm{f}}$ is the input to the system, and the leading vehicle $a_{1}$ the disturbance. The initial condition $\mathbf{x}(0)=\left[\begin{array}{ll}x_{\mathrm{r}, 0} & v_{\mathrm{r}, 0}\end{array}\right]^{T}$ is determined and set as soon as the sensor detects a new lead vehicle that ACC should follow.

In distance control mode, $a_{\mathrm{d}}$ is given by proportional feedback control of the distance separation error $e_{x}=x_{\mathrm{d}}-x_{\mathrm{r}}$ and its derivative $e_{v}=\dot{e}_{x}=v_{\mathrm{d}}-v_{\mathrm{r}}$

$$
a_{\mathrm{d}}=-k_{2} e_{v}-k_{1} e_{x}, \quad k_{1}, k_{2}>0 .
$$

The distance $x_{\mathrm{d}}$ and the feedback gains $k_{i}$ are tuning parameters. In this paper we use a constant spacing control law, where $x_{\mathrm{d}}$ is equal to a constant value $s_{0}=40 \mathrm{~m}, k_{1}=1.2$, and $k_{2}=1.7$. Since the desired relative velocity is obviously equal to zero, (2) can be rewritten as

$$
a_{\mathrm{d}}=k_{2} v_{\mathrm{r}}+k_{1}\left(x_{\mathrm{r}}-s_{0}\right) .
$$

This control law is asymptotically stable, so that both $e_{x}$ en $e_{v}$ are always regulated to zero, provided $k_{1}, k_{2}>0$ (Swaroop, 1994). In this paper we also neglect sensor processing delay and vehicle dynamics by assuming that the desired acceleration is realized at the input of the controlled system without any time lag, such that $u=a_{\mathrm{d}}$. However, we do introduce an actuator saturation, since ACC systems usually restrict the minimum and maximum control input $u$ for safety reasons. In this case study we use the restriction that $a_{\mathrm{f}}$ is bounded between -2.5 and $2.5 \mathrm{~m} / \mathrm{s}^{2}$. 


\subsection{Performance criteria and perturbations for $\mathrm{ACC}$}

The performance of an ACC can be quantified in a number of measures $\rho_{i}$, e.g. overshoot, tracking error, time response, control effort, ride comfort, and string stability. Here we restrict the controller validation to the measure of safety, expressed as the probability $p$ that no collision will occur for a whole range of traffic situations. The safety measure for a single experiment is denoted by $\rho_{\mathrm{s}} \in\{0,1\}$, where $\rho_{\mathrm{s}}=1$ means that the ACC system manages to follow the preceding vehicle at a safe distance, and $\rho_{\mathrm{s}}=0$ means that the traffic scenario would require a brake intervention by the driver to prevent a collision ${ }^{1}$. Depending on the resulting value for $p$, the feedback gains $k_{1}$ and $k_{2}$ can be optimized. In practice, it is not desirable to achieve $p=1$ for all traffic scenarios, since this would necessitate a very conservative controller with high autonomous braking capacity. ACC field test results suggest that $p=0.95$ is more or less appropriate for highway cruising (Fancher et al., 1998).

The value of $\rho_{\mathrm{s}}$ for a particular traffic scenario obviously depends on the perturbations imposed by that scenario. The disturbance to the ACC system is formed by the motion of other vehicles that are detected by the environment sensors. Apart from the acceleration of the preceding vehicle $a_{1}$, also the initial conditions $\mathbf{x}(0)$ determine the probability of a collision. These scenario parameters, together with measurement noise, process noise, unmodelled dynamics and various types of faults construct an $n$ dimensional perturbation space $\Delta$. It is then of interest to evaluate the function $\rho_{\Delta}$ that reflects the dependency of $\rho$ on the structure of this complex and probably nonconvex space $\Delta$. In reality the perturbation space is constructed by dozens of parameters (including sensor characteristics, driver behavior, and vehicle dynamics). In this paper we restrict the validation to $\Delta$ being limited to the subset $\mathscr{S}=\left\{a_{1}, x_{\mathrm{r}, 0}, v_{\mathrm{r}, 0}\right\}$ of uncertain traffic scenario parameters and where the resulting safety measure $\rho_{\mathrm{s}}(\Delta)$ is non-decreasing.

\subsection{The control system validation problem}

Designing a simple stable controller that meets the ACC performance requirements is not difficult. However, validation of this controller with respect to these requirements and subsequent tuning may require a lot of effort. For low-order systems, controller validation can still be solved in a deterministic way or by using iterative algorithms. But when the dimension of $\Delta$ increases and $\rho(\Delta)$ becomes non-convex, it can be expected that the problem will become more difficult to solve, and eventually become intractable, as shown in the tutorial paper by Vidyasagar (1998).

Currently, controller validation is therefore often performed by a grid search, where all parameters are varied through their operating range (see, e.g., Fielding et al. (2002)). However, such a validation strategy is not very efficient, since an exhaustive grid search may require a very large number of experiments, perhaps even too large to be feasible. Alternatively, a worst-case analysis can be performed by lumping together a combination of disturbances, each with the direction and magnitude that has the worst impact on the system performance $\rho$. Such a worst-case analysis on a control system is however unrealistic, since it may result in a conservative controller that is tuned to a specific non-reachable combination of operating conditions. In the next sections we therefore

\footnotetext{
${ }^{1}$ Please note the difference between the performance level $\rho$ for a particular experiment and its probability $p$ for a whole range of experiments.
} 
discuss and further extend an approach to address this problem. 


\section{A randomized algorithm for control system valida- tion}

\subsection{Motivation for a probabilistic approach}

An alternative approach for solving a complex problem exactly, is to solve it approximately by using a randomized algorithm (RA). An RA makes random choices during its execution, and covers both Monte Carlo, sequential and other probabilistic algorithms (Motwani, 1995). The use of an RA can turn an intractable problem into a tractable one, but at the cost that the algorithm may fail to give a correct solution (without the user knowing). The probability that the RA fails can be made arbitrarily close to zero, but never exactly equal to zero. A popular example is a Monte Carlo simulation strategy, where this probability depends on the sample complexity, i.e. the number of simulations performed. An important issue is therefore the necessary sample complexity that guarantees a certain level of confidence for the simulation outcome. In this section we show that this sample complexity is bounded, depending on the desired level of accuracy and reliability, but also that these bounds are rather conservative.

\subsection{Bounded sample complexity for RAs}

The use of a randomized approach for controller validation can be illustrated as follows. Consider an arbitrary process with only two possible outcomes: 'failure' $(\rho=0)$ and 'success' $(\rho=1)$. Suppose that we wish to determine the probability $p$ for a successful outcome of this process. If $N$ denotes the number of experiments with this process and $N_{\mathrm{S}}$ the number of experiments with successful results, then the ratio $N_{\mathrm{S}} / N$ is called the empirical probability or empirical mean $\hat{p}_{N}$ for a successful result of the process.

However, $\hat{p}_{N}$ is unlikely to be exactly equal to the real probability $p$, although it is reasonable to expect that $\hat{p}_{N}$ will approach $p$, as $N \rightarrow \infty$, and as long as the samples are chosen to be representative of the set $\Delta$. The question thus arises in what sense $\hat{p}_{N}$ converges to $p$, and how many experiments $N$ have to be performed to give a reasonable estimate of $p$. An estimate $\hat{p}_{N}$ can be called reasonable if it differs from the real (unknown) value $p$ by no more than $\varepsilon>0$, such that

$$
\left|p-\hat{p}_{N}\right| \leq \varepsilon
$$

where $\varepsilon$ is called the accuracy of the estimate.

Since $\hat{p}_{N}$ is a random variable depending on the particular realization of the $N$ samples, the inequality (4) is only valid with a certain probability of realization. Therefore, we cannot always guarantee that $\left|p-\hat{p}_{N}\right| \leq \varepsilon$, even with a large number of experiments. This means that if the experiments are performed another $N$ times, the estimate $\hat{p}_{N}$ will probably have another value. The probability that $\left|p-\hat{p}_{N_{j}}\right|>\varepsilon$ for a particular set of simulations $N_{j}$ is then the unreliability $\delta$ of the estimate $\hat{p}_{N}$. We would therefore like to know the level of accuracy $\varepsilon$, and reliability $1-\delta$, that can be obtained with one particular set of $N$ simulations. In practice, often the reverse problem is considered: how many experiments $N$ are necessary to achieve a desired level of confidence, in terms of $\varepsilon$ and $\delta$ ?

To answer this question, first define the desired accuracy for determining the unknown quantity $p$ by some number $\varepsilon>0$. Then, in order to know the reliability of our set of experiments, it is necessary to determine the probability that performing $N$ 
Table 1: Values for $\varepsilon, \delta, N_{\mathrm{ch}}$, and $N_{\mathrm{wc}}$, according to the Chernoff bound (5) and the worst-case bound (10).

\begin{tabular}{|l|c|c|r|}
\hline \multicolumn{1}{|c|}{$\boldsymbol{\varepsilon}$} & $\varepsilon$ & $N_{\mathrm{ch}}$ & $N_{\mathrm{wc}}$ \\
\hline 0.10 & 0.10 & 150 & 22 \\
0.05 & 0.10 & 185 & 29 \\
0.03 & 0.10 & 210 & 34 \\
0.02 & 0.10 & 231 & 38 \\
0.01 & 0.10 & 265 & 44 \\
0.002 & 0.10 & 346 & 59 \\
\hline
\end{tabular}

\begin{tabular}{|l|l|r|r|}
\hline \multicolumn{1}{|c|}{$\delta$} & \multicolumn{1}{c|}{$\varepsilon$} & \multicolumn{1}{c|}{$N_{\text {ch }}$} & \multicolumn{1}{c|}{$N_{\text {wc }}$} \\
\hline 0.05 & 0.05 & 738 & 59 \\
0.02 & 0.05 & 922 & 77 \\
0.01 & 0.05 & 1060 & 90 \\
0.02 & 0.03 & 2559 & 129 \\
0.01 & 0.01 & 26492 & 459 \\
0.001 & 0.001 & 3800452 & 6905 \\
\hline
\end{tabular}

experiments will generate an estimate $\hat{p}_{N}$ for which $\left|p-\hat{p}_{N}\right|>\varepsilon$. The Chernoff bound (Chernoff, 1952) states that this probability, defined as $\delta>0$, is no larger than $2 e^{-2 N \varepsilon^{2}}$. This means that, after performing the experiment $N$ times, we can state with a confidence of at least $1-2 e^{-2 N \varepsilon^{2}}$ that the empirical probability $\hat{p}_{N}$ is no more than $\varepsilon$ different from the true but unknown probability $p$. So, in order to estimate the unknown quantity $p$ to an accuracy of $\varepsilon$ and with a confidence of $1-\delta$, the number of experiments $N$ should be chosen such that $2 e^{-2 N \varepsilon^{2}} \leq \delta$, or equivalently

$$
N_{\mathrm{ch}} \geq \frac{1}{2 \varepsilon^{2}} \ln \frac{2}{\delta},
$$

which is known as the additive Chernoff bound. Table 1 presents the necessary sample complexity to calculate $\hat{p}_{N}$ for some values of $\varepsilon$ and $\delta$. Since $\hat{p}_{N}$ has a confidence interval, the upper bound for $N$ is called a soft bound, as opposed to hard bounds given by a deterministic algorithm.

\subsection{Formulation of an RA}

The Chernoff bound forms the basis for the application of Monte Carlo methods, used widely in engineering for design and analysis of control system performance. Several references give a clear tutorial introduction to the use of RAs (Alippi, 2002; Calafiore et al., 2003; Tempo et al., 2004; Vidyasagar, 1998). Here we summarize the main steps to be taken in control system analysis using RAs.

Suppose that the closed-loop system (in our case the ACC equipped vehicle and its controller) must be verified for a certain performance level $\rho$. It is then the goal to estimate the probability $p$ that this performance $\rho$ lies above a pre-specified threshold value $\gamma$. In order to compute ${ }^{2} \hat{p}_{N}(\gamma)$, we generate $N$ independent identically distributed (iid) samples $\Delta_{1}, \Delta_{2}, \ldots, \Delta_{N}$ in the perturbation space $\Delta$ according to its probability density function (pdf) $f_{\Delta}(\Delta)$. The outcome of every $i$-th experiment is represented by an indicator function $J$, given by

$$
J\left(\Delta_{i}\right)= \begin{cases}0, & \text { if } \rho<\gamma \\ 1, & \text { if } \rho \geq \gamma\end{cases}
$$

The empirical probability $\hat{p}_{N}$ can then be estimated as

$$
\hat{p}_{N}=\frac{1}{N} \sum_{i=1}^{N} J\left(\Delta_{i}\right),
$$

\footnotetext{
${ }^{2}$ In the following we will also use $\hat{p}_{N}$ instead of $\hat{p}_{N}(\gamma)$ for reasons of clarity.
} 
which is known as the simple sampling estimator. Since the outcome of the inequality $\left|p-\hat{p}_{N}\right| \leq \varepsilon$ is a random variable, it has a certain probability of realization. By introducing a confidence degree $1-\delta$, this probability is defined as

$$
\operatorname{Pr}\left\{\left|p(\gamma)-\hat{p}_{N}(\gamma)\right| \leq \varepsilon\right\} \geq 1-\delta, \quad \forall \gamma \geq 0, \delta \in(0,1), \varepsilon \in(0,1)
$$

The sample complexity $N$ that is required for (8) to hold, can then be calculated from (5). This method for probabilistic control system analysis is formalized as follows:

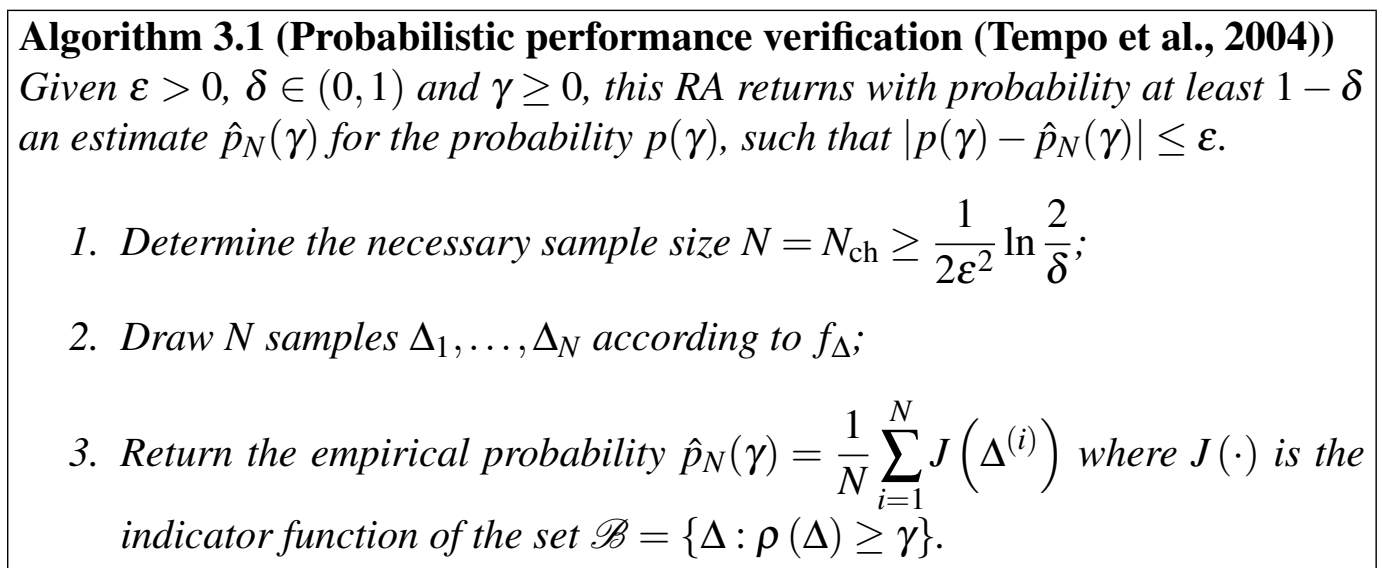

\subsection{Examples of application of an RA}

In this section we will apply Algorithm 3.1 to the simplified ACC case study, of which the 'true' outcome is exactly known. If we are interested in knowing the number of traffic situations that would become dangerous, given a stochastic distribution of traffic scenarios, we can use Algorithm 3.1 to give an estimate $\hat{p}_{N}$ of this probability $p$ with an accuracy level $\varepsilon$ and a confidence level $\delta$, based on $N$ simulations.

\subsubsection{Example 1: Uniformly distributed disturbance}

Scenario definition In this first example we choose a highway cruising scenario where the lead vehicle approaches a traffic jam and brakes to a full stop. The initial conditions are $x_{r, 0}=x_{\mathrm{d}}=40 \mathrm{~m}$, and $v_{1}(0)=v_{\mathrm{f}}(0)=30 \mathrm{~m} / \mathrm{s}$. We assume that the deceleration of the preceding vehicle is the only disturbance with a uniform distribution between -10 and $0 \mathrm{~m} / \mathrm{s}^{2}$, denoted as $f^{\mathrm{U}}\left(a_{1}\right) \sim \mathscr{U}[-10,0]$. We also restrict the analysis to the measure of safety $\rho_{\mathrm{s}}$. In situations when the lead vehicle brakes hard, the ACC vehicle cannot obtain the necessary deceleration $a_{\mathrm{d}}$, since the actuator saturates at $-2.5 \mathrm{~m} / \mathrm{s}^{2}$. Now, for fine-tuning the controller parameters, we would like to know the percentage of brake situations for which $\rho_{\mathrm{s}}$ meets a pre-defined threshold. The threshold $\gamma$ is simply set at 1 in this case (no collision).

The safety of the system obviously decreases with a stronger deceleration $a_{1}$, such that the function $\rho_{\mathrm{s}}\left(a_{1}\right)$ is non-decreasing. Therefore, the boundary value $\Delta_{\gamma}$, for which only just a collision is prevented, can easily be calculated using an iterative algorithm as $-3.015 \mathrm{~m} / \mathrm{s}^{2}$. Below this value the scenario will always result in a collision, above this value the ACC vehicle will be able to stop autonomously and prevent a collision. Since $a_{1}$ is uniformly distributed on the interval $[-10,0]$, the real value for $p$ is thus equal to $(3.015 / 10)=0.3015$. The probability that the estimated value $\hat{p}_{N}$ differs from this real value by an accuracy $\varepsilon$ is then $1-\delta$, as defined by (8). 


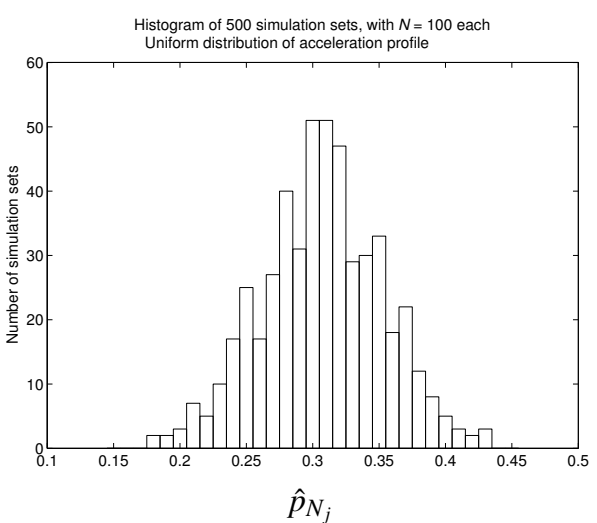

(a) Histogram of 500 simulation sets, with $N=100$ each. where $a_{1} \sim \mathscr{U}(-10.0)$.

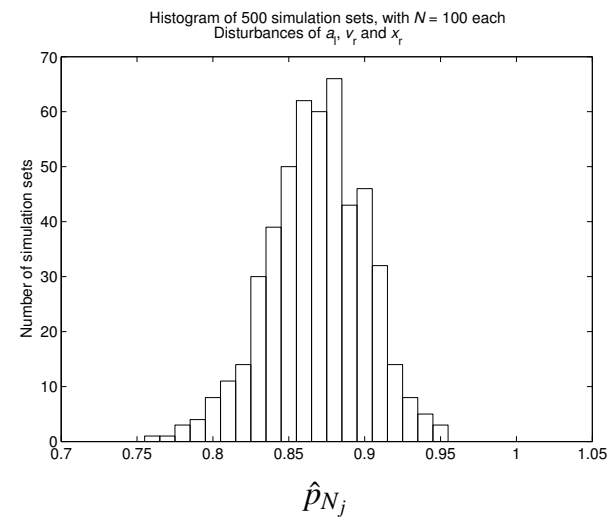

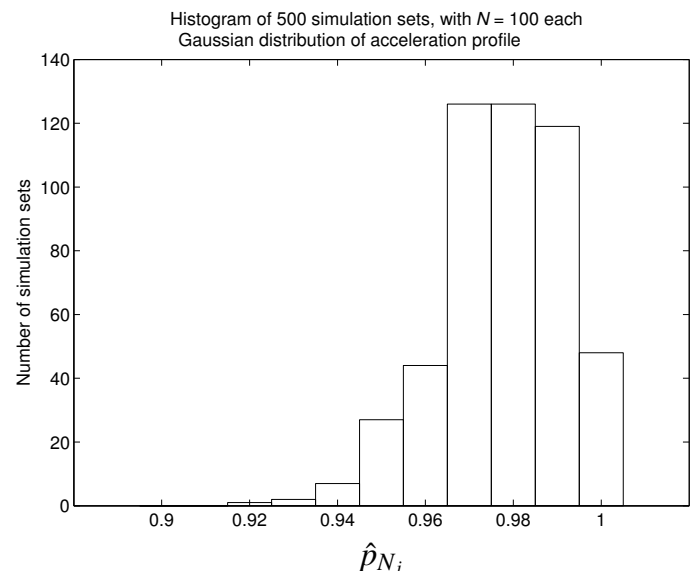

(b) Histogram of 500 simulation sets, with $N=$ 100 each, where the acceleration profile is sampled from a Gaussian distribution $\mathscr{N}(0,1.5)$.

(c) Histogram of 500 simulation sets, with $N=$ 100 each, with a multi-dimensional disturbance for $\mathscr{S}=\left\{v_{\mathrm{l}}, v_{\mathrm{f}}, x_{\mathrm{r}}, a_{\mathrm{l}}\right\}$.

Figure 3: Histograms for the estimate $\hat{p}_{N_{j}}$.

Randomization of the problem Although for this example it is feasible to formulate a deterministic algorithm, in practice it can be difficult or even impossible to determine $p$ in a deterministic way, when the dimension of $\Delta$ increases and the relation $\rho(\Delta)$ becomes non-convex. So instead of calculating $p$ explicitly in deterministic sense, the function is randomized in such a way that it takes a random input $\Delta_{i}$ from its distribution function $f^{\mathrm{U}}\left(a_{1}\right)$, according to Algorithm 3.1.

In order to verify the performance of Algorithm 3.1, we execute it 500 times (each with $N=100$ ). So every $j$-th simulation set, for $j=1, \ldots, 500$, gives us an estimate $\hat{p}_{N_{j}}$. The distribution of the estimate is shown in the histogram in Figure 3(a). With this example, the accuracy and reliability for a single simulation set (each consisting of 100 simulations), can be estimated as $\hat{\varepsilon}$ and $\hat{\delta}$ respectively. The empirical mean $\hat{p}_{M}$ of the probability of a collision-free scenario is 0.30714 , based on all $M N=50000$ simulations, which is very close to $p=0.3015$, as could be expected. The variance of each individual estimate $\hat{p}_{N_{j}}$ can be found by the unbiased estimator for the variance $\sigma_{N_{j}-1}^{2}=\frac{1}{N_{j}-1} \sum_{j=1}^{N_{j}}\left(\hat{p}_{N_{j}}-\hat{p}_{M}\right)$, which gives $\sigma_{N_{j}-1}^{2}=0.0021042$.

Analysis of the simulation results Suppose we desire that $\delta=0.10$ and $\varepsilon=0.10$; (5) then gives $N_{\mathrm{ch}}=150$ as an upper bound on the sample complexity. The desired values 
for $\delta$ and $\varepsilon$ imply that the empirical mean $\hat{p}_{N_{j}}$ for the $j$-th set of simulations should lie in $[0.2015,0.4015]$ for every 9 out 10 simulation sets. However, from Figure 3(a) we observe that in total only 15 out of the 500 simulation sets result in $\hat{p}_{N_{j}} \notin[0.2015,0.4015]$. So $\delta$ is empirically determined at $\hat{\delta}=15 / 500=0.03$, which is smaller than the desired $\delta$. The estimate $\hat{p}_{N_{j}}$ for any simulation set $j$ is thus more reliable than desired, and we only used 100 experiments, whereas (5) requires $N_{\mathrm{ch}}=150$.

This example suggests that a certain value for $\delta$ and $\varepsilon$ can be achieved with a lower number of samples than deemed necessary by the Chernoff bound. Correspondingly, by choosing $N=N_{\text {ch }}$, a higher level of accuracy and reliability can be obtained. Although the degree of conservatism for this example is rather limited, it can be shown that this conservatism increases with smaller values for $\delta$ and $\varepsilon$ (Vidyasagar, 1998).

\subsubsection{Example 2: Gaussian Distributed Disturbance}

In the previous example, $f^{\mathrm{U}}\left(a_{1}\right)$ may not be representative for the entire operating range, whereas $\hat{p}_{N}$ greatly depends on the correctness of the underlying pdf. Let us therefore assume a more general acceleration profile for highway cruising. Typical vehicle measurements during ACC field testing (Fancher et al. (1998)) suggest that the acceleration profile can be roughly described as a random signal with a Gaussian distribution $f^{\mathrm{N}}$ with mean $\mu=0$ and standard deviation $\sigma=1.5$, denoted as $\mathscr{N}(0,1.5)$, truncated on the interval $[-10,10] \mathrm{m} / \mathrm{s}^{2}$.

We now execute Algorithm 3.1 again with $a_{1} \sim \mathscr{N}(0,1.5)$. Figure $3(\mathrm{~b})$ shows the resulting histogram with the estimated collision probability $\hat{p}_{N_{j}}$ for every simulation set is shown. The empirical mean $\hat{p}_{M}$ is now 0.97752 , with a variance of $\sigma_{N_{j}-1}^{2}=$ 0.00020506 , which is much smaller than in Example 1, due to the lower occurrence rate of dangerous situations.

Suppose that $\delta=0.02$ and $\varepsilon=0.03$ are desired; (5) then gives $N_{\mathrm{ch}}=2559$. From Figure $3(\mathrm{~b})$ we see that only 10 out of 500 estimates $\hat{p}_{N_{j}}$ fall outside the interval $[p-$ $\varepsilon, p+\varepsilon]$, which gives an estimated reliability $\hat{\delta}=0.02$. So the same level of accuracy and reliability has been achieved with only $N=100$ instead of $N_{\mathrm{ch}}=2559$ samples.

\subsubsection{Example 3: Multi-dimensional disturbance}

In the previous two examples $a_{1}$ was considered the only disturbance. However, in practice $p$ depends also on the initial distance and velocities of both vehicles at the moment of first detection by the sensor. Consider for instance a cut-in situation at close distance $x_{\mathrm{r}}$, which is more dangerous than a vehicle cutting-in at larger distance (with identical $a_{1}$ and $v_{\mathrm{r}}$ ). The crash probability thus depends on the entire set $\mathscr{S}=$ $\left\{v_{1}, v_{\mathrm{f}}, x_{\mathrm{r}}, a_{1}\right\}$ that defines the longitudinal scenario, as discussed in Section 2.2. In this example we assume Gaussian distributions for both the velocities (with $\mu=30 \mathrm{~m} / \mathrm{s}$ and $\sigma=5 \mathrm{~m} / \mathrm{s}$ ), and distance $x_{\mathrm{r}}$ (with $\mu=60 \mathrm{~m}, \sigma=20 \mathrm{~m}$, and truncated on $[10,150] \mathrm{m}$ ).

Figure 3(c) shows the resulting histogram after execution of Algorithm 3.1 with

$\hat{p}_{M}=0.86848$ and $\sigma_{N_{j}-1}^{2}=0.0010378$. Compared to Example 2 it can be seen that the variance is much larger, since the outcome of the experiment now depends on four independent pdfs. The sample complexity $N$ is again conservative, since Figure 3(c) shows that only one estimate falls outside the interval $[p-\varepsilon, p+\varepsilon]$, indicating $\hat{\delta}=$ 0.002 . So the same level of accuracy and reliability has been achieved with only $N=$ 100 instead of $N_{\mathrm{ch}}=346$ samples. 


\subsection{Characteristics properties of RAs}

The examples and key references (Alippi, 2002; Calafiore et al., 2003; Tempo et al., 2004; Vidyasagar, 1998) point out the following characteristic properties:

- A limited number of test runs is necessary as compared to deterministic algorithms. However, the sample complexity to achieve a reasonable $\varepsilon$ and $\delta$, as given by the Chernoff bound, is quite conservative.

- An RA is very simple, since the Chernoff bound is completely independent of the nature of the underlying process and the perturbation space $\Delta$. However, this also means that no advantage is taken of any a priori knowledge of the structure of $\Delta$. It is therefore desired to modify the simulation approach such that knowledge of the system is applied.

- The reliability of the simulation outcome strongly depends on the reliability of the pre-defined $\operatorname{pdf} f_{\Delta}$. The outcome of the simulation approach also greatly depends on the modeling effort.

- The empirical mean $\hat{p}_{N}$ does not say anything about the minimum or maximum level of performance that can be expected. It may well be that a control system has a good average performance, but also a poor worst-case performance.

In the next section methods used in conventional control system validation will be applied to address these issues. These methods will be integrated with the RA approach, and illustrated with the ACC case study. The resulting methodology will be presented in Section 5. 


\section{Reduction of the sample complexity}

As mentioned in the previous section, the sample complexity $N$ given by the Chernoff bound is too conservative. Reduction of $N$ for ADAS validation is therefore an important challenge. One way is to reformulate the problem and use a different test objective. In addition, the sampling space $\Delta$ can be reduced by neglecting certain subsets that are impossible to occur. Subsequently, $N$ can be further reduced by using a priori knowledge on which samples will be more interesting than others. These reduction techniques are also valid for conventional validation techniques, but are now integrated with the RA approach.

\subsection{A reduced worst-case bound}

The examples in Section 3 were based on estimation of the mean performance $p$. Alternatively, we can estimate the worst-case performance $\rho_{\max }$ by $\hat{\rho}_{N}=\max _{i=1,2, \ldots, N} \rho\left(\Delta_{i}\right)$. Tempo et al. (1997) prove that the minimum number of samples $N$ which guarantees that

$$
\operatorname{Pr}\left\{\operatorname{Pr}\left\{\rho(\Delta)>\hat{\rho}_{N}\right\} \leq \varepsilon\right\} \geq 1-\delta
$$

is then given by

$$
N \geq \frac{\ln 1 / \delta}{\ln 1 /(1-\varepsilon)}
$$

The sample complexity given by (10) is much lower than for (5), as shown in Table 1, and is more suitable in case of a worst-case analysis.

\subsection{Reduction of the sampling space}

Figure 4(a) shows a scatter plot with the occurrence of collisions in the perturbation space $\Delta$ for Example 3. Obviously, a collision is more likely with lower values for $x_{\mathrm{r}, 0}$, $v_{\mathrm{r}, 0}$, and $a_{\mathrm{l}}$, such that the collision occurrences are clustered in a specific subspace $\Delta_{\mathrm{F}}$.
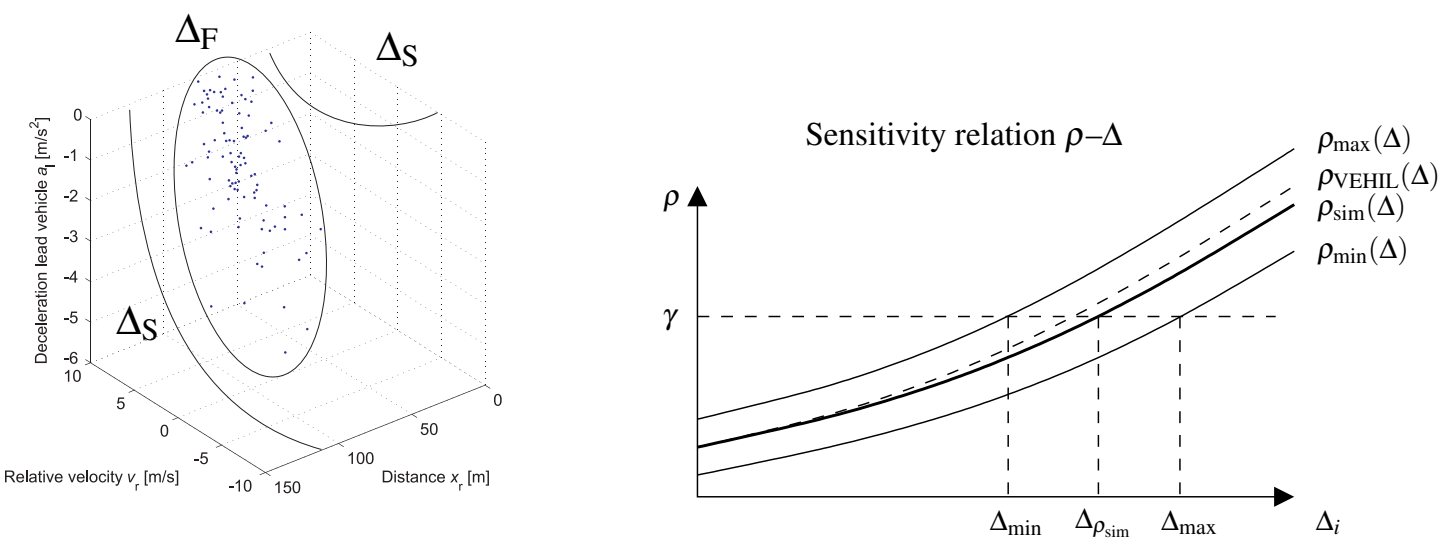

(a) Scatter plot with the occurrence of collisions(b) Dependency between the performance characterFurthermore, the subspaces $\Delta_{\mathrm{S}}$ and $\Delta_{\mathrm{F}}$ of which thestic $\rho$ and the specific value of $\Delta$. outcome can be a priori established as success or failures.

Figure 4: Illustration of why and how the sample space could be reduced. 
This means that there is structure in the perturbation space $\Delta$ and in the function $\rho(\Delta)$ that can be used to reduce the necessary perturbation space of interest. The sampling space can then be reduced by disregarding specific subsets of $\Delta$, of which the outcome is a priori known. An example is the subset $\Delta_{\mathrm{S}}$ with combinations of positive acceleration and positive relative velocity that will never result in a potential collision.

When this dependency $\rho(\Delta)$ can be proven to be convex or non-decreasing, the sample complexity $N$ can be significantly reduced, as illustrated in Figure 4(b). In this case an RA can iteratively search for the boundary value $\rho\left(\Delta_{\gamma}\right)=\gamma$, and neglect any $\Delta_{i}$ larger than $\Delta_{\gamma}$, since the outcome of those samples can be predicted in advance.

\subsection{Importance sampling}

As stated above, it makes sense to give more attention to operating conditions that are more likely to cause a collision than others.

Suppose that we want to estimate a probability $p$, given a perturbation $\Delta$. If $f_{\Delta}$ is a pdf $\sim \mathscr{U}[0,1]$ on the interval $S=[0,1]$, our goal is then to estimate

$$
p=\int_{S} J(\Delta) f_{\Delta}(\Delta) d \Delta=E[J(\Delta)]
$$

where $J$ is the performance function, and $\Delta \sim f_{\Delta}$. Importance sampling is a sampling technique to increase the number of occurrences of the event of which the probability $p$ should be estimated (Madras, 2002). This estimation is corrected by dividing it by the increased probability of the occurrence of the event. In order to highlight the interesting subset $\Delta_{\mathrm{F}}$ it thus makes sense not to sample from the original pdf $f_{\Delta}$, but instead use an artificial pdf, reflecting the importance of the events, and then reweighing the observations to get an unbiased estimate.

We can now define an importance sampling pdf $\varphi$ that is strictly positive on $S$. We can then write

$$
p=\int_{S}\left(\frac{J(\Delta) f_{\Delta}(\Delta)}{\varphi(\Delta)}\right) \varphi(\Delta) d \Delta=E\left[\frac{J(\Phi) f_{\Delta}(\Phi)}{\varphi(\Phi)}\right]
$$

where $\Phi \sim \varphi$. The importance sampling estimator based on $\varphi$ is

$$
\hat{p}[\varphi]_{N}=\frac{1}{N} \sum_{i=1}^{N} \frac{J\left(\Phi_{i}\right) f_{\Delta}\left(\Phi_{i}\right)}{\varphi\left(\Phi_{i}\right)}
$$

where $\Phi_{1}, \ldots, \Phi_{N}$ are iid with pdf $\varphi$. Its variance is

$$
\operatorname{var}\left(\hat{p}[\varphi]_{N}\right)=\frac{1}{N}\left[\int_{S} \frac{J(\Delta)^{2} f_{\Delta}(\Delta)^{2}}{\varphi(\Delta)} d \Delta-p^{2}\right]
$$

An efficient estimator $\hat{p}[\varphi]_{N}$ is obtained by choosing $\varphi$ proportional to the importance' of the individual samples, where importance is defined as $\left|J(\Delta) f_{\Delta}(\Delta)\right|$. A rare but dangerous event can thus be equally important as a more frequent but less dangerous event. 


\section{Methodological approach}

A major problem with ADAS controller validation is that the system cannot be tested $e x$ haustively for every disturbance under every operating condition. A validation methodology should therefore provide a suitable test program in order to sufficiently (but also efficiently) cover the entire perturbation space. To this aim we propose a generic methodological approach, consisting of the steps described in the following sections.

\subsection{Specification}

Firstly, define performance measures $\rho$ and corresponding evaluation criterion $\gamma$. In addition, the desired $\delta$ and $\varepsilon$ must be defined. Correspondingly, select the test objective in order to determine the type of bound for $N$ :

- Probability of performance satisfaction: for a given $\delta$, $\varepsilon$, check if the performance measure $\rho$ is below threshold $\gamma$ with a certain probability level $p$ for the whole perturbation space $\Delta$.

- Worst-case performance: check if the worst-case performance $\rho_{\max }$ is within $\varepsilon$ of $\hat{\rho}_{N}$ with a certain probability $1-\delta$.

Then, identify the perturbation space $\Delta$ and its pdf $f_{\Delta}$ by using preliminary field test results. Using knowledge on the structure of $\Delta$ or the function $\rho(\Delta)$, determine subsets $\Delta_{\mathrm{F}}$ and $\Delta_{\mathrm{S}}$, of which the outcome is a priori known (either failure or success). Furthermore, a simulation model of the vehicle, its sensor system, and its control system is designed using the dedicated simulation tool PRESCAN (Labibes et al. (2003)).

\subsection{Simulation}

Then execute an RA using importance sampling (cf. Section 4.3) to cover the important part of the perturbation space to estimate the performance $\hat{p}_{N}$ with respect to the criteria defined earlier. In general $\rho$ can be a continuous value, although we used a discrete value in the examples.

The performance of an RA using importance sampling depends heavily on the reliability of the models and the pdfs used in the simulation phase. The robustness of $\hat{p}_{N}$ to model uncertainty should therefore be considered when validating an ADAS in a randomized approach. The experimental relation between $\rho$ and $\Delta$ from the simulations is then bounded between $\rho_{\max }$ and $\rho_{\min }$, as illustrated in Figure 4(b). This means that the estimated boundary value lies within the interval $\left[\Delta_{\min }, \Delta_{\max }\right]$, provided that $\rho(\Delta)$ is a non-decreasing relation.

\subsection{Model validation}

Therefore, the most interesting samples of the perturbation space $\Delta_{i}$ are chosen to be reproduced in the VEHIL facility, also in a randomized approach to efficiently cover $\Delta$. These particular $\Delta_{i}$ are selected to lie within the interval $\left[\Delta_{\min }, \Delta_{\max }\right]$. In VEHIL disturbances can be introduced in a controlled and accurate way, thereby achieving a more reliable estimate than $\rho_{\text {sim }}(\Delta)$. In this way the model uncertainty can be reduced, 
because of the replacement of a vehicle and sensor model by real hardware. The corresponding test program can be formalized as follows:

\section{Algorithm 5.1 (Probabilistic model validation)}

1. Choose initial values for $\Delta_{\min }$ and $\Delta_{\max }$ in accordance with the uncertainty size, and a suitable $N$.

2. Test $\rho$ at $\Delta_{\min }$

3. IF $\rho \geq \gamma$, THEN decrease $\Delta_{\min }$ and GOTO 2 .

IF $\rho<\gamma$, THEN decrease $\Delta_{\max }$ and GOTO 4 .

4. Test $\rho$ at $\Delta_{\max }$

5. IF $\rho \geq \gamma$, THEN increase $\Delta_{\min }$ and GOTO 2 .

IF $\rho<\gamma$, THEN increase $\Delta_{\max }$ and GOTO 4 .

6. Return the empirical probability

$$
\hat{p}_{N}=\frac{1}{N} \sum_{i=1}^{N} J\left(\Delta^{(i)}\right)
$$

The sample complexity $N$ for VEHIL is calculated as follows. Suppose that the requirement is that $\rho \leq \gamma$, where $\gamma$ is a small number, and we want to determine $N$ in the case that all tests have been successful, i.e. $\hat{p}_{N}=1$. Then we want to check if $\operatorname{Pr}\left\{\left|\hat{p}_{N}-p\right| \leq \varepsilon\right\} \geq 1-\delta$, where $\varepsilon$ is set equal to $\gamma$. From $\varepsilon$ and the desired confidence level $\delta$, the necessary number $N$ can then be derived with the Chernoff bound.

Apart from obtaining $\rho_{\text {VEHIL }}$, the test results can also be used for model validation. The estimate $\hat{p}_{N}$ may indicate necessary improvements in the system design regarding fine-tuning of the controller parameters. Improve the simulation model using the VEHIL test results until the simulation model proves to provide adequate performance, convergence in $\hat{p}_{N}$ and sufficient samples $N$.

\subsection{Performance measure}

In an iterative process the simulation results in step 5.2 and thus the estimate $\hat{p}_{N}$ can be improved. Subsequently, the VEHIL test program in step 5.3 can be better optimized by choosing a smaller interval $\left[\Delta_{\min }, \Delta_{\max }\right]$. From the combination of simulation and VEHIL results the performance $\hat{p}_{N}$ of the ADAS can then be estimated with a high level of reliability, and the controller design can be improved. Finally, determine $\rho_{\mathrm{VEHIL}}$ with (13), correcting it for the higher occurrence rate in the interval $\left[\Delta_{\min }, \Delta_{\max }\right]$. 


\section{Conclusions}

We have presented a methodological approach for probabilistic performance validation of advanced driver assistance systems, and applied a randomized algorithm (RA) to a simple adaptive cruise control problem. This probabilistic approach cannot prove that the system is safe or reliable. However, we accept a certain risk of failure (though small), since any other conventional validation process (e.g. test drives) is also based on a probabilistic analysis. Furthermore, use can be made of a priori information on the system, thereby emphasizing interesting samples.

Ongoing research is focused on extension of this methodological approach to more complex ADAS models, with non-convex performance functions $\rho_{\Delta}$ and multiple performance criteria (safety, stability, and driving comfort). Furthermore, model validation will be performed using a real hardware setup in the VEHIL facility. 


\section{Acknowledgments}

Research supported by TNO Automotive, TRAIL Research School, and the TU Delft spearhead program “Transport Research Centre Delft: Towards Reliable Mobility”. 


\section{References}

Alippi, C. (2002) Randomized algorithms: A system-level, poly-time analysis of robust computation, IEEE Trans. on Computers, 51(7), pp. 740-749.

Calafiore, G., F. Dabbene, R. Tempo (2003) Randomized algorithms in robust control, in: Proc. of the 42nd IEEE Conference on Decision and Control, Maui, Hawaii, USA, pp. 1908-1913.

Chernoff, H. (1952) A measure of asymptotic efficiency for tests of a hypothesis based on the sum of observations, Annals of Mathematical Statistics, 23, pp. 493-507.

Fancher, P., R. Ervin, J. Sayer, M. Hagan, S. Bogard, Z. Bareket, M. Mefford, J. Haugen (1998) Intelligent cruise control field operational test, Tech. Rep. DOT HS 808 849, DOT/National Highway Traffic Safety Administration, final report.

Fielding, C., A. Vargas, S. Bennani, M. Selier, eds. (2002) Advanced Techniques for Clearance of Flight Control Laws, Springer, Berlin, Germany.

Gietelink, O., D. Verburg, K. Labibes, A. Oostendorp (2004) Pre-crash system validation with PRESCAN and VEHIL, in: Proc. of the IEEE Intelligent Vehicles Symposium (IV), Parma, Italy.

Labibes, K., Z. Papp, A. Thean, P. Lemmen, M. Dorrepaal, F. Leneman (2003) An integrated design and validation environment for intelligent vehicle safety systems (IVSS), in: Proc. of the 10th World Congress on Intelligent Transport Systems and Services (ITS), Madrid, Spain, paper 2731.

Madras, N. (2002) Lectures on Monte Carlo Methods, American Mathematical Society, Providence, Rhode Island, USA.

Motwani, R. (1995) Randomized Algorithms, Cambridge University Press, New York.

Swaroop, D. (1994) String Stability of Interconnected Systems: An Application to Platooning in Automated Highway Systems, Ph.D. thesis, University of California at Berkeley.

Tempo, R., E. Bai, F. Dabbene (1997) Probabilistic robustness analysis: Explicit bounds for the minimum number of samples, Systems \& Control Letters, 30, pp. 237-242.

Tempo, R., G. Calafiore, F. Dabbene (2004) Randomized Algorithms for Analysis and Control of Uncertain Systems, Springer-Verlag, Berlin, Germany.

Vidyasagar, M. (1998) Statistical learning theory and randomized algorithms for control, IEEE Control Systems Magazine, 18(6), pp. 69-85. 